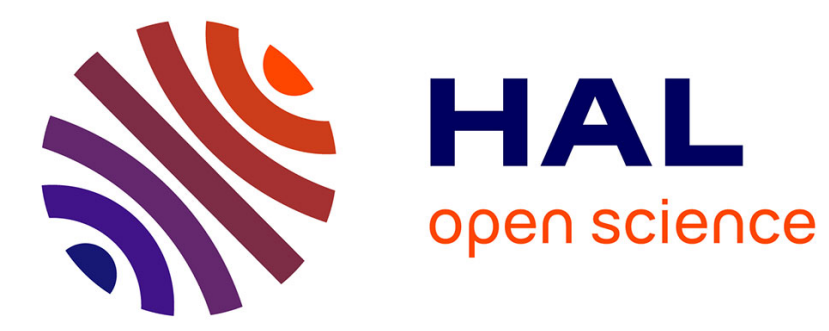

\title{
How Topological Rearrangements and Liquid Fraction Control Liquid Foam Stability
}

\author{
A.-L. Biance, Aline Delbos, Olivier Pitois
}

\section{To cite this version:}

A.-L. Biance, Aline Delbos, Olivier Pitois. How Topological Rearrangements and Liquid Fraction Control Liquid Foam Stability. Physical Review Letters, 2011, 106, pp.068301. 10.1103/PhysRevLett.106.068301 . hal-00790447

\section{HAL Id: hal-00790447 \\ https://hal.science/hal-00790447}

Submitted on 1 Sep 2015

HAL is a multi-disciplinary open access archive for the deposit and dissemination of scientific research documents, whether they are published or not. The documents may come from teaching and research institutions in France or abroad, or from public or private research centers.
L'archive ouverte pluridisciplinaire HAL, est destinée au dépôt et à la diffusion de documents scientifiques de niveau recherche, publiés ou non, émanant des établissements d'enseignement et de recherche français ou étrangers, des laboratoires publics ou privés. 


\title{
How Topological Rearrangements and Liquid Fraction Control Liquid Foam Stability
}

\author{
Anne-Laure Biance, ${ }^{1}$ Aline Delbos, ${ }^{2}$ and Olivier Pitois ${ }^{2}$ \\ ${ }^{1}$ Université de Lyon, F-69000, France; Univ. Lyon 1, Laboratoire PMCN; CNRS, UMR 5586; F-69622 Villeurbanne Cedex, France \\ ${ }^{2}$ Université Paris-Est, Laboratoire de Physique des Matériaux Divisés et des Interfaces, FRE 3300 du CNRS, 5, boulevard Descartes, \\ Champs-sur-Marne, 77454 Marne-la-Vallée Cedex 2, France
}

(Received 12 May 2010; published 9 February 2011)

\begin{abstract}
The stability of foam is investigated experimentally through coalescence events. Instability (coalescence) occurs when the system is submitted to external perturbations (T1) and when the liquid amount in the film network is below a critical value. Microscopically, transient thick films are observed during film rearrangements. Film rupture, with coalescence and eventual collapse of the foam, occurs when the available local liquid amount is too small for transient films to be formed. Similar experiments and results are shown in the two-bubble case.
\end{abstract}

DOI: 10.1103/PhysRevLett.106.068301

PACS numbers: 82.70.Rr, 47.55.dk, 83.50.Lh

Diphasic media are out of equilibrium because of their large interfacial energy. Hence, preventing their collapse is a crucial point as shown by the recent development of new highly stable systems [1]. In foams and emulsions, stability is governed by coarsening [2] and coalescence [3]. Coalescence has been studied through its consequences as daughter bubble formations [4], dynamics of droplet fusion [5], or avalanches during foam collapse [6,7]. However, its origin remains poorly understood although it is a crucial point in many fundamental, industrial, and everyday life applications.

Foam can be viewed as a network of thin films separating two contacting gas bubbles. Films meet three by three and form liquid channels with curved interfaces, the socalled Plateau borders (PBs). The films are known to experience capillary suction from the PBs, which induces film thinning, film rupture, and finally foam collapse. Isolated thin film stability is evaluated by the value of a critical disjoining pressure, $\Pi_{\text {crit }}$, that accounts for interactions of the film's interfaces [8]. Though evidence has been provided for correlation between the stabilities of isolated films and foams as reviewed in [3,9], a robust link between $\Pi_{\text {crit }}$ and the conditions of foam collapse is still missing. Recent work [10] suggests that foam stability is affected by spontaneous topological rearrangements inside the foam, the so-called T1 events [11] described in Fig. 1. Studies of T1s have concerned essentially kinetics $[12,13]$ and their statistical distribution in space and time $[14,15]$, in relation to foam rheology. To our knowledge, no study of T1s is focused on stability aspects.

In this Letter, the stability of films involved in bubble rearrangements is shown to be crucial to understand foam stability. The rupture behavior of the film created during a $\mathrm{T} 1$ event limits the overall stability of the system. Rupture occurs if a minimum amount of liquid is not present locally in the foam. In this context, the stability of a macroscopic $3 \mathrm{D}$ foam is investigated by artificially triggering $\mathrm{T} 1 \mathrm{~s}$ and a comparison with an experiment at the scale of two bubbles is performed.

A dedicated foam experiment allows us to probe locally foam stability in causing T1 events. The foam is made in a Perspex tube of height $60 \mathrm{~cm}$ and cross-sectional area $25 \mathrm{~cm}^{2}$ by slowly injecting perfluorohexane saturated nitrogen gas into an aqueous surfactant solution with a syringe needle. Foam with monodisperse bubbles of radius $R$ in the range $0.3-1.5 \mathrm{~mm}$ and made from various foaming solutions reported in Table I are studied. Once the foam has filled the tube, it is let to drain freely, protected by a glass cover. At a given column height, liquid fraction, $\epsilon(t)$, slowly decreases as a function of time. $\epsilon(t)$ is followed in measuring the evolution for the PBs' width $w$ at the column wall: $\epsilon(t) / \epsilon_{0}=w^{2}(t) / w_{0}^{2}$ following the procedure described in [17]. An entry point along the column wall is used to introduce a thin glass capillary into the foam sample, and to perfuse a small amount of gas in order to cause T1 events. The volume perfused is approximately 5 times the bubble volume, and the perfusion lasts 5 seconds. Gas is perfused when the liquid fraction $\epsilon(t)$ reaches the desired value. As gas is perfused, film rupture and coalescence events are detected by video techniques. Such a

TABLE I. Foaming solution compositions: TTAB (3 g/l), LOH (0.3 g/1), SLES (0.3 g/1, Stepan Co.), CAPB (0.15 g/1, Goldschmidt), MAc (0.02 g/1)- $\epsilon_{\text {crit }}$ measured in macroscopic foam $(*)$ and bubble cluster $(* *)$ - ratio of elongational viscosity $\kappa$ versus surface tension. Elongational viscosity was measured by the oscillating bubble method (deformation $10 \%, 0.1 \mathrm{~Hz}$ ). Mixing procedure is described in [16].

\begin{tabular}{lcccccc}
\hline \hline & Composition & $\epsilon_{\text {crit }}^{*}$ & $\epsilon_{\text {crit }}^{* *}$ & $\frac{\kappa}{\gamma}(\mathrm{ms}) \tau^{*}(\mathrm{~ms}) \tau^{* *}(\mathrm{~ms})$ \\
\hline A & TTAB & $8 \times 10^{-4}$ & $10^{-3}$ & 3 & 1.5 & 0.9 \\
B & TTAB,LOH & $<10^{-4}$ & $10^{-4}$ & 1800 & 765 & 1500 \\
C & SLES,CPAB & $1.3 \times 10^{-4}$ & $3 \times 10^{-4}$ & 30 & 13 & 10 \\
DSLES,CPAB,MAc & $<10^{-4}$ & $10^{-4}$ & 3000 & 1000 & 2000 \\
\hline \hline
\end{tabular}




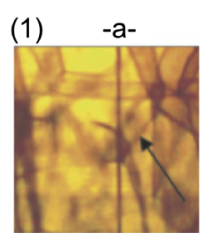

(2)
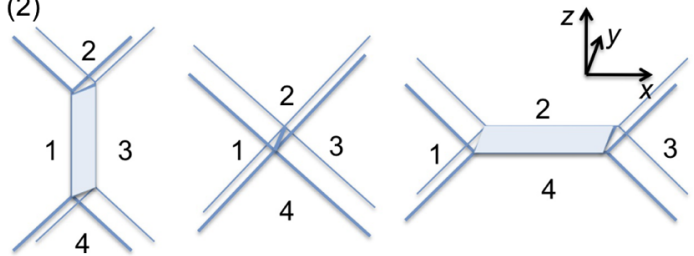

FIG. 1 (color online). (1) Image sequence showing one T1 event in a 3D foam made of TTAB solution (A) at a liquid fraction $\epsilon>\epsilon_{\text {crit }}$. Images (a), (b) and (c) correspond to situations, respectively, before, just after, and long after bubble rearrangement. Color pattern in (b) reveals the thickening of the new film formed by $\mathrm{T} 1$. The size of images is approximately $1.5 \mathrm{~mm}$. (2) Scheme of the corresponding T1 rearrangement.

measurement is performed for several bubble sizes and liquid fractions. This procedure allows us to distinguish values of $\epsilon$ where film rupture events are observed within the perfusion area from those where bubbles rearrange without coalescence. The value corresponding to the transition is referred to as the critical liquid fraction, $\epsilon_{\text {crit }}$. Note that coalescence events caused within the perfusion area generally induce avalanches of $\mathrm{T} 1 \mathrm{~s}$ and numerous film rupture events occur throughout the foam sample (foam collapse).

Results obtained for $\epsilon_{\text {crit }}$ of foams made with the solutions are reported as a function of bubble radius in Fig. 2. There is no obvious dependence of $\epsilon_{\text {crit }}$ on $R$, and the average value measured over the full range of bubble radii is $\epsilon_{\text {crit }} \approx 0.0008 \pm 0.0004$, in good agreement with [10].

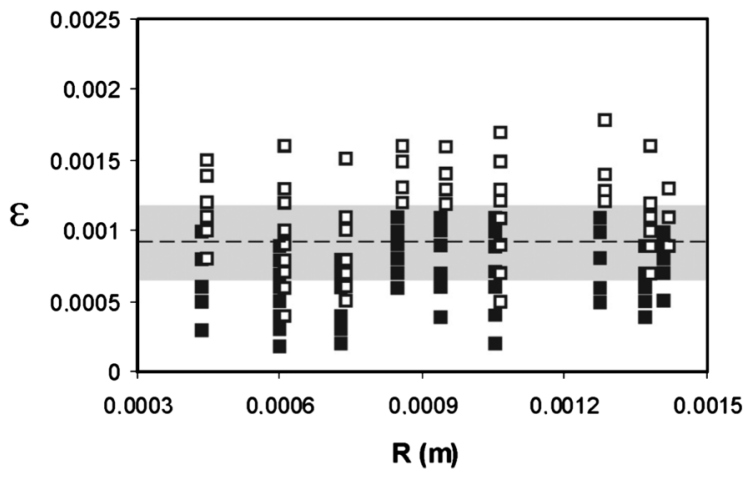

FIG. 2. Diagram showing stability behavior of foam bubbles during T1 events as a function of foam liquid fraction $(\epsilon)$ and bubble radius $R$ : unstable events (filled squares), stable events (open squares). Liquid fraction range for transition between stable and unstable events is marked by the grey area and the critical liquid fraction is defined as the median value in this range (dashed line).
With solution B and D (rigid surfactant), such a quantitative result is not obtained because corresponding values for the critical liquid fraction are very low and reach the limits of the measurement method. Nevertheless, during experiments performed with solution B we have not observed a rupture event caused by gas perfusion for liquid fractions $\epsilon>10^{-4}$, so that we deduce that $\epsilon_{\text {crit }} \leq 10^{-4}$. The value for mobile solutions are by far larger than that predicted by theory based on the films' disjoining pressure. The balance of liquid pressures in films and $\mathrm{PBs}$ implies: $\Pi \simeq \gamma / r$, where $\gamma$ is the surface tension and $r$ is the radius of curvature of PBs as depicted in Fig. 3(2). The macroscopic liquid fraction in foam can be related to the geometry of PBs through: $\epsilon \simeq r^{2} / 3 R^{2}$ [11], involving that $\epsilon_{\text {crit }} \simeq$ $\gamma^{2} / 3 R^{2} \Pi_{\text {crit. }}^{2}$. $\Pi_{\text {crit }}$ is of the order of $3 \times 10^{4} \mathrm{~Pa}$ for solution $\mathrm{A}$ [18], which gives $\epsilon_{\text {crit }} \sim 10^{-6}$ in the range of investigated bubble radii compared to $8 \times 10^{-4}$ observed experimentally. A sequence of images showing the behavior of films created during T1s, in the case $\epsilon>\epsilon_{\text {crit }}$, is reported in Fig. 1 and reveals a strong thickening of the new film created during the T1 event. Foam films are thin (black films) before and after bubble rearrangement, but during the rearrangement, the forming film is clearly thicker, as indicated by colored patterns.

Observations in three-dimensional foam samples do not allow us to study thoroughly the stability criterion in dynamical events. A simpler experimental configuration is studied, with only two bubbles. As presented in Fig. 3, two soap films are deposited at the ends of two vertical cylindrical tubes (outside diameter between 1 to $10 \mathrm{~mm}$ ), facing each other and held at a distance controlled with a micrometer screw. Connections of the tubes to a syringe pump allow us to inject air and thus to inflate two bubbles of controlled radius $R$ measured by videoscopy before bubbles come into contact. After contact, the two bubbles are joined by a thin liquid film surrounded by a circular PB, as described in Fig. 3. The quantity of liquid in this ring is related to the radius of curvature $r$ of the PB and to the radius of separating contact film, $R_{c}: V \approx 2 \pi R_{c} \delta r^{2}$, where $\delta=3\left(\frac{\pi}{6}-\frac{1}{\sqrt{3}}\right)$ is a geometrical constant, in the limit of $r \ll$ $R_{c}$. In order to reproduce the conditions of very low liquid content experienced by foam close to $\epsilon_{\text {crit }}$, it is then necessary to remove liquid from the system. This is achieved as follows: bubbles perform a run of contact-separation
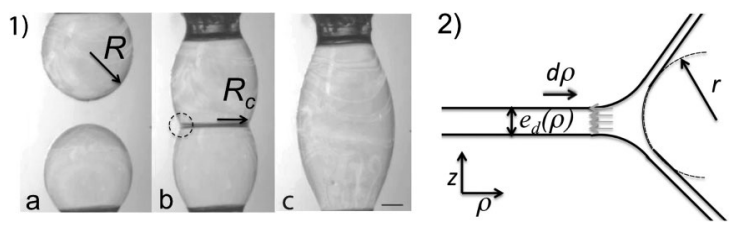

FIG. 3. (1) Picture of two bubbles before contact (a), in contact (b), after a coalescence (c). Scalebar is $3 \mathrm{~mm}$. (2) Scheme of the Plateau border linking bubbles in the two-bubble cluster encircled in (1b). 


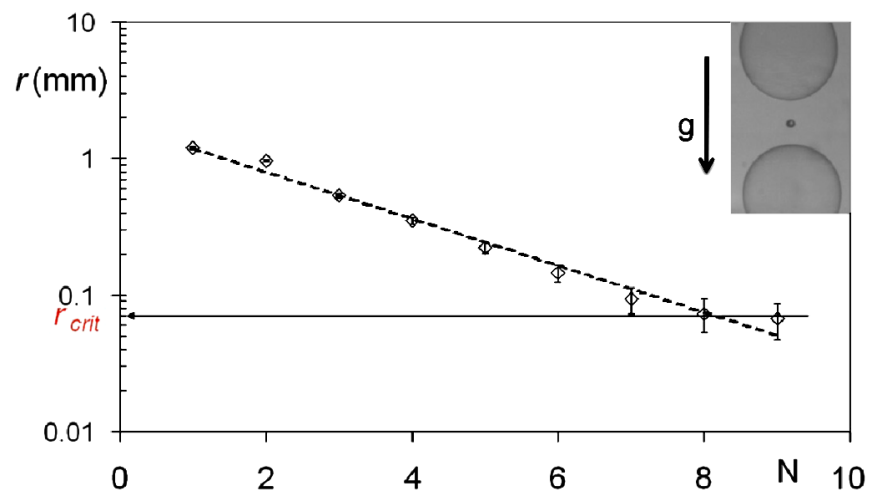

FIG. 4 (color online). Radius of curvature of the Plateau border $r$ linking two bubbles versus the number of contact $N$ in a semilog plot. The solution used is solution D. Bubble radius is $3 \mathrm{~mm}$. The line fits $r=1.75 e^{-0.39 N}$. Inset: bubbles $1 \mathrm{~ms}$ after separation. $(R=2.3 \mathrm{~mm})$.

cycles. Because a satellite droplet is ejected at each separation stage (as depicted on Fig. 4), $r$ and $V$ are decreasing functions of $N$. The evolution of $r$ as a function of $N$ is reported in Fig. 4, showing that $r(N)$ is reasonably described by an exponential function. We estimate from the fit parameter that $45 \%$ of the liquid present in the PB is lost at each separation stage. Figure 4 reveals also the existence of a critical value for the PB radius of curvature, $r_{\text {crit }}$, below which the transient film cannot form during contact stage and coalescence is observed. Experiments conducted on a large number of bubble radii with the different soap solutions listed in Table I have evidenced that this behavior is a general feature of soap films: stable bubble contact dynamic is observed only if the volume of liquid available in the PB is larger than the critical value. Figure 5 presents values obtained for $r_{\text {crit }}$ as a function of $R$.

To link these results with macroscopic foam experiments, it is worth estimating the corresponding critical liquid fraction in the two-bubble experiment. The macroscopic liquid fraction in foam can be related to the

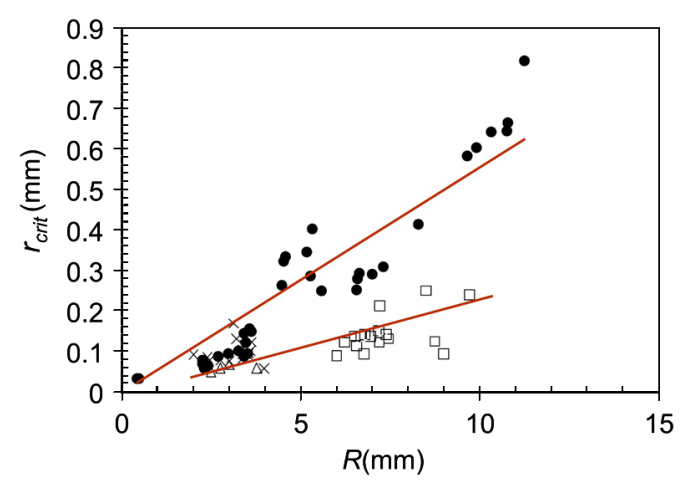

FIG. 5 (color online). Radius of curvature of the Plateau border $r$ at the last contact before coalescence versus bubble radius $R$. Solution A $(\bigcirc)$, solution $\mathrm{B}(\square)$, solution $\mathrm{C}(\times)$, solution $\mathrm{D}(\triangle)$. The red lines correspond to linear fit $r=p R$ with $p=0.056(\bigcirc)$ and $p=0.019(\square)$. geometry of $\mathrm{PBs}$ through $\epsilon_{\text {crit }} \approx 0.33 r_{\text {crit }}^{2} / R^{2}$ [11]. This criteria is used for the two-bubble case. The variations of $r_{\text {crit }}$ versus $R$, reported in Fig. 5, shows a linear dependence, indicating that again a constant critical liquid fraction control the coalescence of the bubbles. The linear approximation $(r=p R)$ reported in Fig. 5 allows us to estimate this critical liquid fraction, given by $\epsilon_{\text {crit }}=$ $0.33 p^{2}$ and reported in Table I. The results obtained are in good agreement with experiments in the macroscopic foam. This suggests that results on both foam and twobubble scales can be understood with a unique mechanism, based on a critical volume of liquid available in PBs for creating an entire new film. Indeed, when the bubbles come into contact, a liquid connection is formed between them, which extends radially because of surface minimization and a film is created at the center. This liquid connection is then at the interception of three films and is a so-called PB.

During film extension, liquid in the PB is supplied by the initial bubble films (static ones) and withdrawn by the contact film formed dynamically, in a similar way to the 3D foam. If the amount of liquid is not sufficient in the PB, the new film cannot be created and coalescence occurs. This has been confirmed by observations: as $\epsilon>\epsilon_{\text {crit }}$, a freshly formed thick film is created between the two bubbles (colored patterns), and as $\epsilon<\epsilon_{\text {crit }}$, the transient film breaks at the edge, close to connected PBs.

A model of this mechanism is proposed for the $3 \mathrm{D}$ foam as for the two-bubble experiments. The mechanism of film creation from the PB is exactly similar, provided the geometry to consider (radial geometry in the two-bubble experiments, 1D geometry in macroscopic foam). In the plane perpendicular to the freshly formed film $[(x, z)$ in Fig. 1 and $(\rho, z)$ in Fig. 3], a new film of length $L$ is formed in foam by the symmetrical receding of $\mathrm{PB}(\mathrm{s})$. The liquid volume conservation in $\mathrm{PB}$ and forming film entails in both cases:

$$
e_{d}(\rho) d \rho \approx-2 \delta r(\rho) d r
$$

where $d \rho$ is an elementary horizontal displacement, $e_{d}(\rho)$ is the local thickness of the new film and $d r$ is the elementary variation of the PB radius of curvature. In 3D foam, local volume conservation can be applied although PBs are connected. Indeed, at the T1 time scale, liquid transport through adjacent PBs is not efficient [19]. There is no available general theory to express $e_{d}(\rho)$ in our configuration, where a film forms from a PB of finite volume within conditions of a varying pulling force. However, the order of magnitude of $e_{d}(\rho)$ can be estimated from Frankel's law [20] with constant receding velocity, $L / \tau$. This law remains valid in the two-bubble case providing that $\rho \gg r$. It reads:

$$
e_{d}(\rho) \approx 1.9 r(\rho)\left(\frac{\eta L / \tau}{\gamma}\right)^{2 / 3}
$$

Introducing Eq. (2) in Eq. (1), $r_{\text {crit }} \approx 1.9 L(\eta L / \tau \gamma)^{2 / 3} / 4 \delta$, and the expected critical liquid fraction of collapse reads 


$$
\epsilon_{\text {crit }} \approx\left(\frac{1.9 \beta}{4 \delta \sqrt{3}}\right)^{2}\left(\frac{\eta \beta R}{\tau \gamma}\right)^{4 / 3}
$$

where $\beta=L / R \approx 0.7$ (two-bubble experiment; $R_{c} / R \approx$ 0.82). Typical values for $\tau$ are obtained from the duration of $\mathrm{T} 1$ events measured during experiments, given by $R / \tau \sim 10^{-1} \mathrm{~m} / \mathrm{s}$ for solution $\mathrm{A}$ and $R / \tau \sim 5 \times$ $10^{-3} \mathrm{~m} / \mathrm{s}$ for solution $\mathrm{B}$, in agreement with those published in $[12,13]$ with similar solutions. (In the two-bubble experiments, the value of $\tau^{* *}$ are deduced from measurements of the variations of $R_{c}$ with time, just above the critical liquid fraction and are in good agreement.) Values predicted by Eq. (3) are thus respectively $\epsilon_{\text {crit }} \sim 5 \times 10^{-4}$ and $\epsilon_{\text {crit }} \sim 2 \times 10^{-5}$, which falls directly in the respective ranges of values measured for the critical liquid fraction of foam collapse. The corresponding film average thicknesses $e$ can be estimated from measured values for $\epsilon_{\text {crit }}: e \gtrsim$ $(6 \delta / \beta) R \epsilon_{\text {crit }}$, yielding values in the range $1-10 \mu \mathrm{m}$ for solution A, which is consistent with color patterns observed during foam experiment. To this regard, the duration of $\mathrm{T} 1$ events appears to be a relevant parameter in this problem and thus introduces in $\epsilon_{\text {crit }}$ a strong dependance on interfacial rheology that sets the velocity of film creation. Recent work on the dynamics of $\mathrm{T} 1$ events indicates that $\tau \sim \kappa / \gamma$, where $\kappa$ is the dilatational viscosity of the interface [13]. Values reported in Table I for $\kappa / \gamma$ show that $\epsilon_{\text {crit }}$ is controlled by interfacial viscosity, which corroborate the proposed mechanism for foam destabilization. The latter is therefore a starting point to understand the key role of dynamic properties of the interfaces (elasticity and surface viscosity) on foam stability, reported in many experiments since the 1950s [3,9,21]. But this naive model has to be extended to predict more accurately the experimental results. For example, measured values of $\epsilon_{\text {crit }}$ do not demonstrate the bubble radius dependence predicted by Eq. (3). Obviously, this reflects that Frankel's law does not accurately account for the complex interfacial phenomena in T1 events. As recently highlighted [22], the dependence of the film thickness with meniscus size involves coupling terms with surface parameters that remain to be understood thoroughly. However that may be, Eq. (3) opens a new way in the understanding of $\epsilon_{\text {crit }}$.

To conclude, the stability of foams is significantly affected by dynamical events. During bubble rearrangements, liquid amount available locally can be insufficient to create a new film. This has been clearly attributed to a dynamical thickening effect of the fresh film, whose thickness is strongly dependent on the rheological properties of interfaces. The proposed simple model captures these new findings and shows good quantitative agreement with our measurements for the critical liquid fraction of foam collapse. This new coupling between the T1s' dynamics and foam stability opens a new way of understanding avalanche mechanisms described in foam collapse [6,7], and their correlation with $\mathrm{T} 1$ avalanches in foam rheology [15].

[1] M. Abkarian, A. B. Subramaniam, S.-H. Kim, R. J. Larsen, S.-M. Yang, and H. A. Stone, Phys. Rev. Lett. 99, 188301 (2007).

[2] J. Lambert, R. Mokso, I. Cantat, P. Cloetens, J. Glazier, F. Graner, and R. Delannay, Phys. Rev. Lett. 104, 248304 (2010).

[3] D. Langevin, Adv. Colloid Interface Sci. 88, 209 (2000).

[4] J. Bird, R. de Ruiter, L. Courbin, and H. A. Stone, Nature (London) 465, 759 (2010).

[5] S. Thoroddsen, K. Takehara, and T. Etoh, J. Fluid Mech. 527, 85 (2005).

[6] H. Ritacco, F. Kiefer, and D. Langevin, Phys. Rev. Lett. 98, 244501 (2007).

[7] N. Vandewalle, J. F. Lentz, S. Dorbolo, and F. Brisbois, Phys. Rev. Lett. 86, 179 (2001).

[8] A. Sheludko, Adv. Colloid Interface Sci. 1, 391 (1967).

[9] L. E. Scriven, Chem. Eng. Sci. 12, 98 (1960).

[10] V. Carrier and A. Colin, Langmuir 19, 4535 (2003).

[11] D. Weaire and S. Hutzler, The Physics of Foams (Clarendon Press, Oxford, 1999).

[12] M. Durand and H. A. Stone, Phys. Rev. Lett. 97, 226101 (2006).

[13] A.-L. Biance, S. Cohen-Addad, and R. Hohler, Soft Matter 5, 4672 (2009).

[14] I. Cantat and O. Pitois, Phys. Fluids 18, 083302 (2006).

[15] A. Kabla and G. Debregeas, Phys. Rev. Lett. 90, 258303 (2003).

[16] K. Golemanov, N.D. Denkov, S. Tcholakova, M. Vethamuthu, and A. Lips, Langmuir 24, 9956 (2008).

[17] S. A. Koehler, S. Hilgenfeldt, and H. A. Stone, Langmuir 16, 6327 (2000).

[18] V. Bergeron, Langmuir 13, 3474 (1997).

[19] O. Pitois, C. Fritz, and M. Vignes-Adler, J. Colloid Interface Sci. 282, 458 (2005).

[20] K. J. Mysels and S. P. Frankel, J. Colloid Interface Sci. 66, 166 (1978).

[21] V. Craig, Current Opinion in Colloid \& Interface Science 9, 178 (2004).

[22] E. A. van Nierop, B. Scheid, and H. A. Stone, J. Fluid Mech. 602, 119 (2008). 